\title{
介质各向异性和非均匀性对横波分裂参数 测量的影响
}

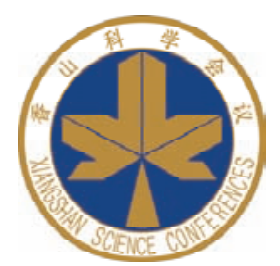

雷军

北京大学地球与空间科学学院, 北京 100871

E-mail: leij@pku.edu.cn

2017-02-08 收稿, 2017-03-06 修回, 2017-03-07 接受, 2017-05-08 网络版发表

国家自然科学基金(41674053)资助

摘要地震横波分裂参数是确定地球介质各向异性基本特征的重要依据. 在过去几十年间, 快横波偏振方向和 快-慢横波到时差测量值的显著离散已经成为影响学界对地震各向异性和横波分裂进行深入研究的一个突出障碍. 本文具体分析了实际地球介质的各向异性和非均匀性可能引起的地震记录上地震波震相观测走时和理论走时的 偏离、观测偏振和理论偏振的偏离, 以及震相观测序列和理论序列的偏离; 指出了实际三分量地震记录上地震波 震相矢量普遍存在的偏振非正交和路径非正交关系是传统正交分离和参数测量结果显著离散的根本原因; 用仿 真模拟实例分析了现有震相识别和波形分析方法可能带来的震相漏识别或误识别, 介绍了利用矢量分析技术分离 三分量观测记录上地震波震相完整波形的基本思路, 推导了以地震波空间偏振矢量方向为参数的波形分离运算表 达式，以及直接利用三分量地震记录的傅里叶频谱在不同坐标分量上的投影来测量地震记录上各个震相矢量空间 方向的近似方法.

关键词横波分裂，各向异性，非均匀性，非正交，矢量

从 20 世纪 80 年代到今天, 地震各向异性的研究 在全球范围内取得了长足的进展. 英国爱丁堡大学 的Crampin ${ }^{[1 \sim 6]}$ 确立了地震横波分裂现象与介质各向 异性的内在物理关系, 将横波分裂确定为地球介质 各向异性的重要标志, 预示了地震各向异性研究在 了解地球内部物理过程中的广阔应用. 地震学家随之 在世界各地开展的大量的地震各向异性和横波分裂观 测研究揭示了各向异性广泛存在于地壳 ${ }^{[7 ~ 17]}$ 、地幔和 核幔边界 ${ }^{[18 \sim 26]}$ 及地核 ${ }^{[27 ~ 29]}$ 等各个分层. 并在理论上证 明了横波分裂参数与地球内部不同深度应力场方向、 大小及其随时间变化的对应关系，一定程度上展示了 在地震孕育、发生前后地球内部应力场调整、变化的 图像, 成为地震物理预报有迹可循的重要线索 ${ }^{[30 \sim 34]}$.
地震横波观测还揭示出地壳浅部岩石中孔隙、裂隙的 张开与闭合与油气的储藏、运移的关联 ${ }^{[35 ~ 40]}$.

地震横波分裂参数包括分裂横波中快横波偏振 方向和快-慢横波到时差. 其中, 快横波偏振方向与 形成地震各向异性和产生横波分裂现象的地球内部 应力场方向相关, 除断裂带附近外, 通常与观测台站 下部区域压应力主轴方向平行. 依据某个地区不同 观测点之间快波偏振方向及其差异可构建出该地区 应力状态的空间方向分布; 快-慢横波到时差反映了 在上述应力场作用下地球介质弹性特征的变化和偏 离各向同性的程度, 是地球内部现今应力大小、介质 特征和介质应力响应的直接显示 ${ }^{[41 ~ 47]}$. 经过地震学 家数十年的努力, 分裂横波中快波偏振的观测结果 
已经得到了整个地球科学领域同行的广泛认同, 在 一定的精度上与全球或区域构造运动方向的其他观 测结果相互映证. 但是, 有关各向异性介质中快-慢 横波到时差的观测和解释并没有显示足够的 “客观

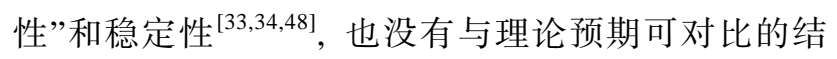
果. 到目前为止, 没有一幅清晰、简明的图像可以显 示横波的快-慢波到时差与地球内部运动或地震孕育 过程中应力的积累和释放过程具有无可争辩的依赖 关系 ${ }^{[49]}$. 尽管这种依赖关系在理论和物理因果上都 是合理、必然的 ${ }^{[2 \sim 4,7,11,50 \sim 54]}$. 事实上, 大量观测数据 显示横波到时差测量结果十分离散, 无论在地壳还 是在地幔 ${ }^{[55,56]}$ 的观测中都存在接近一个数量级的偏 差, 即使在一个范围有限的地区也是这样. 横波分裂 到时差的观测和解释已经陷人困境 ${ }^{[47,48]}$, 严重制约 了通过横波分裂观测更深人地了解与地震各向异性相 关联的地球内部运动及演化. 为了找出造成横波分裂 参数测量困难的主要原因, 必须重新梳理介质各向异 性对地震横波运动学和动力学特征的影响 ${ }^{[57]}$, 重新认 识地震横波在更接近真实地球介质的地震各向异性介 质中与其在均匀各向同性理想介质中的差别, 以及这 些差别和影响在三分量观测记录上的具体体现.

\section{1 非理想地球介质中地震横波的基本特征}

各向异性介质比均匀各向同性介质更精细、更接 近真实地球介质. 物理上各向异性是介质微观结构 和弹性特征受地球内部运动或不同温压影响形成的 一种具有方位优势的现象. 在地壳中主要表现为脆 性岩石中孔隙、裂隙在最小主压应力方向的张开和在 最大主压应力方向的闭合所呈现的优势取向. 地幔 中表现为岩石晶格在地幔运动驱使、变形作用下的定 向排列. 地震横波对各向异性介质敏感, 横波进人各 向异性介质不仅发生分裂、其偏振方向也显著改变并 形成非正交, 使各向异性介质条件下地震记录上横 波的基本特征显著不同于各向同性介质, 造成现有 地震波震相分析的正交前提不再满足, 从而影响了 横波分裂参数测量. 与此同时, 地球内部介质的速度 分层或其他非均匀结构引起的纵横波传播路径和震 相序列的改变也对横波分裂参数的测量形成干扰.

\section{1 各向异性介质中地震波传播特征与理想介质 的差异}

介质各向异性从根本上改变了均匀各向同性理
想介质条件下地震纵横波的运动学和动力学特征 ${ }^{[4]}$, 主要体现在：(1) 发生类似于物理光学中的双折射现 象, 横波分裂为快横波 $\mathrm{qS} 1$ 和慢横波 $\mathrm{qS} 2$, 同时地震 纵横波速度也随其传播方向与介质各向异性对称面 的关系而变. (2) 纵横波偏振特征变化 ${ }^{[58 \sim 60]}$. 在各向 异性介质中, 当地震波传播方向既不平行也不垂直 于各向异性对称面时纵波的偏振方向将不再与传播 方向平行, 形成准纵波 $\mathrm{qP}$; 横波偏振也不再与人射 面或垂直人射面关联, 而是受介质各向异性对称面 约束. 快横波偏振将与各向异性椭圆长轴方向平行. (3) 三类体波的相互耦合. 除各向异性对称面外, 各 向异性介质中三类体波在界面上相互耦合 ${ }^{[3,7]}$, 不存 在独立的、与纵波解耦的 $\mathrm{SH}$ 型偏振波. 三类体波的耦 合给地震各向异性介质条件下的地震波场增添了比 各向同性更多的复杂性.

这些改变不仅仅带来纵横波观测走时与理论走 时的偏离, 更导致地震记录上纵横波观测偏振与理 论偏振的偏离.

\section{2 各向异性介质条件下横波偏振非正交关系}

偏振是地震波震相最重要的特征, 每一个地震 波震相都是具有特定空间偏振方向的矢量(为强调地 震波震相的偏振矢量特征以下将地震波震相称为“震 相矢量”). 各向同性介质中震相矢量相互正交, 与之 相比各向异性介质条件下震相矢量关系最显著的差 异是相互非正交.

$\mathrm{Li}$ 和Crampin ${ }^{[61]}$ 研究了各向异性介质中分裂横波 偏振的非正交问题，指出了各向异性介质条件下快慢横波偏振存在的“固有非正交”和“视非正交”[60 62]. 其中, 固有非正交与介质的各向异性程度相关, 并随 地震波人射角而变化. 较大的横波固有非正交可能 接近 $20^{\circ}$; 在各向异性介质中快-慢横波偏振正交是非 常偶然的. 只有当地震波射线路径正好与各向异性 对称面平行或正交时才能可能发生 ${ }^{[63]}$, 这要求震源、 介质和接收台站正好位于某个特定方向, 在天然地 震观测中这种巧合是极少发生的. 视非正交与地震 波人射平面和观测坐标平面的空间关系有关，这种 非正交在三分量观测记录上同样不能忽视 ${ }^{[62]}$.

各向异性介质中横波偏振显著偏离各向同性介 质条件，同时纵波偏振也因介质的各向异性程度、人 射方向与介质各向异性对称面的空间关系而不同， 相比之下纵波偏离各向同性的程度小于横波 ${ }^{[3,58,59]}$. 


\section{3 分层及一般非均匀介质中纵横波路径非正交 关系}

三分量观测记录上，地震波震相矢量的非正交 关系并不是介质各向异性地震问题中特有的现象. 各向异性介质中震相矢量的非正交现象主要表现在 同一路径上三类体波的偏振非正交. 事实上, 另一类 地震波震相矢量非正交现象一直以来就存在于地震 记录中, 这就是分层及一般非均匀介质中由于地震 波路径差异形成的“路径”非正交.

无论是均匀各向同性分层介质还是一般横向非 均匀介质, 由于地震波在各个分层界面的反射、折 射、衍射或转换必然形成不同路径和不同偏振方向的 地震波震相矢量以非正交方式在某个时间窗内先后 到达观测台站, 并以矢量方式叠加干涉形成复杂的 三分量矢量观测记录. 事实上, 纵横波正交偏振关系 只有在无限半空间、均匀各向同性介质条件下才是存 在的. 与偏振非正交相比, 震相矢量的路径非正交现 象更加广泛.

实际地震记录上介质各向异性和非均匀性造成 的偏振非正交和路径非正交共同存在, 是三分量地 震记录上提取横波分裂参数、特别是快-慢横波到时 差最大的障碍.

并非只有横波分裂参数的测量才受到地震波震 相矢量非正交问题的困扰. 其实, 除初至震相外几乎 所有地震波震相矢量参数的测量都受到非正交关系 的影响. 以往地震观测数据处理中一个潜在的假定 是地震波震相因路径差异使它们在走时上彼此分开, 从而可将震相矢量之间的非正交问题忽略. 其实, 这 些认识主要来源于对比较简单的速度连续递增的分 层介质模型的正演模拟结果. 地球内部由于介质密 度、温度、压力等状态的差异在不同深度形成不同规 模的所谓介质各向异性和非均匀性分层, 不仅引起 地震波偏振特征的改变, 同时也引起地震波速度、走 时和震相先后次序的改变, 如高速或低速层(界面)走 时曲线上出现的地震波“回折”及非正交“交叉”现象, 直接改变了某些时间窗内震相到达的“正常”先后序 列. 这种现象不仅在近场, 而且在全球地震走时曲线 上也广泛存在. 路径非正交还广泛出现在可控源勘 探数据上, 包括经常出现的测线外反射进入测线形 成的 “三维”干扰问题. 介质各向异性和非均匀性引 起的地震波路径变化、速度变化改变了某些观测时间 窗内震相到达原有的“正常”先后序列，可能使路径、
偏振相差较大的地震波在同一个较小的时间窗内相 遇，从而使非正交问题在不同尺度的地震观测中广 泛存在.

路径非正交在三分量地震记录上最终也表现为 地震波震相矢量的偏振非正交, 成为影响地震波参 数测量最显著的干扰因素. 毫无疑问, 只有在地震各 向异性研究和横波分裂参数测量中地震波震相矢量 的非正交问题才如此突出. 大量横波观测数据显示 纵波尾波对横波快波到时和偏振方向测量的干扰往 往难以简单消除。与此同时，由于快-慢横波在时间 窗内如此靠近, 纵波尾波及快横波都对慢横波带来 影响更是严重阻碍了慢横波到时的可靠辨认和快-慢 横波到时差的有效测量.

真实地球介质中地震波的特征显然比已经被理 论简化的各向异性和非均匀性更为复杂. 事实上, 实 际三分量地震观测记录上无可避免地同时包含了偏 振非正交和路径非正交的共同影响. 与此同时, 震相 矢量之间的非正交关系也携带或映射出比理想介质 更多、更精细的真实介质信息. 只要我们试图更可靠 地测量横波分裂参数, 更可靠地测量其他二次震相、 后续震相和弱震相的参数, 从而实现对介质特征和 地层结构更精细的解析, 就不可能回避各向异性和 非均匀性带来的非正交问题. 这些信息是进一步解 析地球所不可缺少的. 反之, 一旦非正交问题被忽略 就不可能提取到这些震相和介质的可靠信息.

在目前的震相分析和测量方法中, 没有任何判 据可以帮助我们预先判定观测记录上不存在各向异 性或横向非均匀性的影响, 也无法区分各向异性或 横向非均匀性的影响大小和比例.

\section{2 现有地震波震相识别和参数测量存在的 问题}

如前所述, 地球介质的各向异性引起的地震波 速度变化将改变地震波到时的理论预期、偏振非正交 变化将改变地震波偏振的理论预期，加上三类体波 在界面的耦合效应将增加地震波场的复杂性, 并进 一步改变地震波序列的理论预期. 同样, 介质的分 层、非均匀性也会改变地震波到时、路径和震相序列 的理论预期. 无论在近场还是远场, 这些改变可能在 地震记录上的某个时间窗内形成理论预期之外的多 个震相矢量的到达和非正交叠加, 从而影响到三分 量地震记录上地震波震相的识别和参数的测量. 为 
此, 需要基于地震波震相的矢量特征进一步加以分 析和说明.

\section{1 正交波场分离的多解性}

为了减少横波分裂参数测量结果的离散性, 多 年来地震学家发展了多种横波震相分析和参数测量 方法. 但大多数方法的基础依然是基于均匀各向同 性介质假定建立的地震波正交分离技术, 忽略了实 际观测记录上地震波震相的矢量特征和广泛存在的 “非正交”关系.

由于地震波震相的非正交关系, 正交分离得到 的3个分量只是地震波矢量场中多个震相矢量在这些 方向上的部分投影. 这些投影不仅不能代表任何一 个独立的震相矢量, 而且丢失了地震波震相矢量完 整的振幅、相位和波形等动力学信息. 试图利用滤波 或其他的时频分析等标量信号处理技术从这些分量 中可靠恢复已经丢失的地震矢量信息也是不可能的.

数学上如果不同震相矢量之间存在非正交关系, 那么正交分离技术就不可能准确测量各个震相的到 时、振幅、相位和波形等运动学和动力学参数. 包括 横波分裂在内的后续震相以及其他反映介质精细特 征的弱震相、二次转换震相信息都将严重丢失, 必然 产生不可预测的多解性和不确定性. 除某些特例外, 正交分离结果只在一定程度上反映强震相的部分特 征, 只能给出有关地球内部结构的一级近似. 目前, 横波分裂参数的测量已经遇到了不可逾越的障碍 ${ }^{[36]}$.

\section{2 观测记录上地震矢量波波形的不确定性}

各向异性和非均匀性引起的地震波偏振、速度或 到时改变最直观的表现是给观测记录上的地震波形 带来显著的不确定性. 为便于说明, 将震相矢量的空 间方向分别用该震相的偏振人射角 $\theta$ 和偏振方位角 $\varphi$ 来表示, 标注为 $(\theta, \varphi)$, 它与正交观测坐标系的空间 关系如图1所示. 为简化方位角和人射角的标记, 全 文只标注角度的大小值而省略“度”的符号.

图2显示了偏振变化对观测波形和参数测量可能 带来的影响. 图2(a1)中从上到下是3个空间偏振方向 分别为 $(5,40),(18,7)$ 和 $(23,23)$ 的非正交震相矢量, 其波形分别用“蓝色”、“红色”和“绿色”表示 (为简明起 见选择了接近谐波的波形), 并用对应颜色的垂线虚 线分别标注了上述 3 个震相矢量到达观测台站的时 间. 图2(b1) 是图2(a1)中所有震相的合成矢量在观

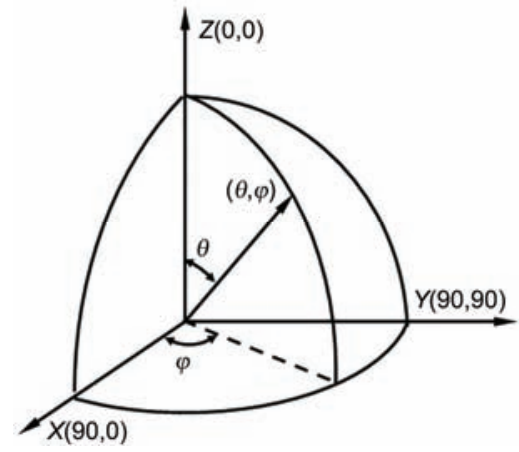

图 1 震相矢量偏振方向与正交观测坐标系示意图. $\theta$, 偏振人射角; $\varphi$, 偏振方位角; 坐标轴 $X, Y$ 和 $Z$ 的方向分别用方向矢量 $(90,0),(90$, $90)$ 和 $(0,0)$ 来表示

Figure 1 Definition of the direction of a polarization vector in Cartesian coordinate system. $\theta$, incident polarizing angle; $\varphi$, azimuth polarizing angle; the direction of axis $X, Y$, and $Z$ are represented by direction vector $(90,0),(90,90)$ and $(0,0)$, respectively

测坐标南北 $(90,0)$, 东西 $(90,90)$ 和垂直 $(0,0)$ 中的 3 个 分量. 图2(b1)与 (a1)中 3 个原始震相差异显著, 显示 记录波形及其振幅与原始震相的波形和振幅不再一 致，这不仅依赖坐标系的选择，同时也依赖传播介质 的特性.

如前所述，与均匀各向同性介质相比各向异性 或非均匀性会改变震相偏振和走时的理论预期, 将 直接影响地震观测记录波形. 首先分析偏振引起的 波形变化, 将图 2(a1)的 3 个震相中第 1 个初至波震相 矢量 $(5,40)$ 的偏振人射角从 5 调整到 25 , 所有震相的 波形及其他参数均保持不变, 形成图 2(a2)及与之对 应的3个观测分量如图2(b2). 对比图2(b2)与(b1)显示 单一震相的偏振变化引起了 3 个观测分量上波形和周 期在整个时间段上的明显改变. 其次再看看走时引 起的波形改变, 将图2(a2)中第2个震相矢量 $(18,7)$ 的 到时延长 3 个时间采样点 $(0.03 \mathrm{~s}$, 本图中数据采样频 率为 $100.0 \mathrm{~Hz}$ )形成图 2(a3), 同样所有震相的其他参 数保持不变, 其3个观测分量如图2(b3). 对比图2(b2) 和(b3)显示观测记录波形差异较大，观测波形随地震 波到时的变化同样敏感. 由此可见，在保持原始震相 波形不变的条件下，介质各向异性或非均匀性对观 测波形影响显著且难以预测. 这些影响, 既不可能通 过简单的坐标变换来消除，也破坏了仅仅通过波形 的某种“特征化”来识别震相的基础.

\section{3 传统方法可能带来的震相误识别或漏识别}

对图2的上述分析显示, 偏振方向以及到时变化 

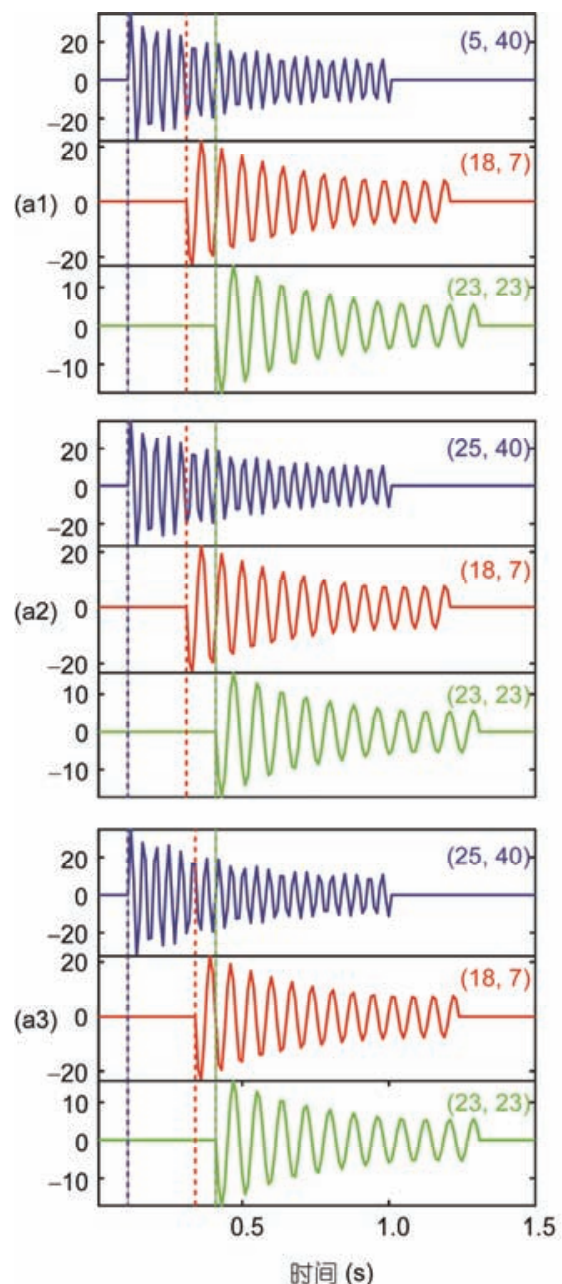
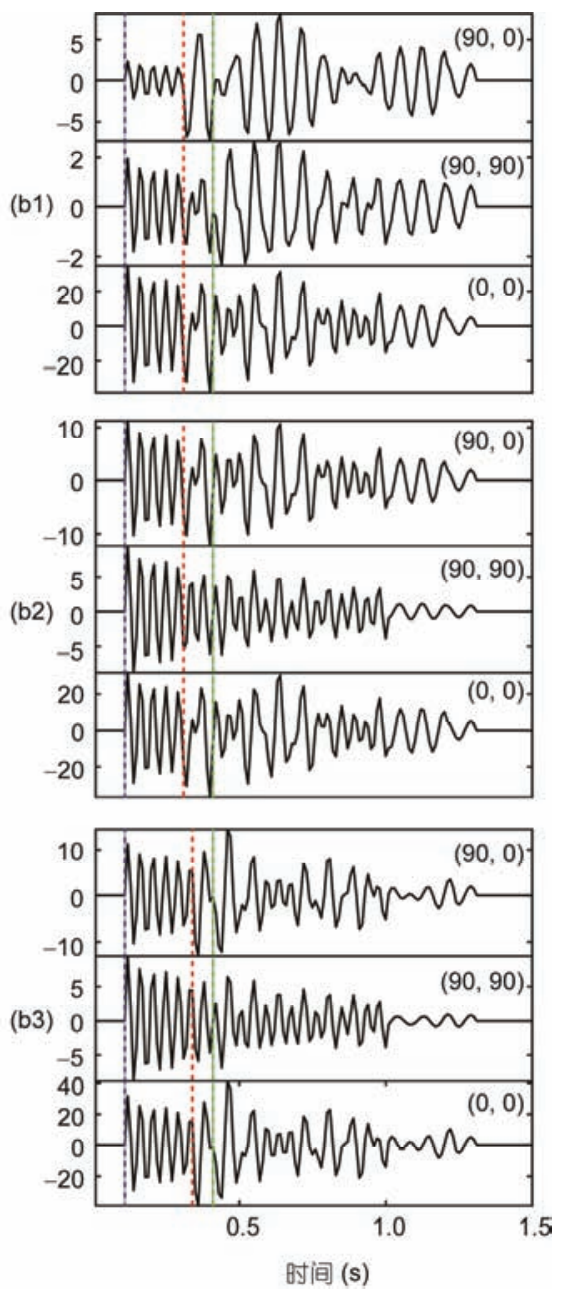

图 2 地震波震相矢量偏振和到时变化对三分量正交观测记录波形的影响. (a1) 偏振方向分别为 $(5,40)$ (蓝线), (18, 7)(红线)和(23, 23)(绿线)的 3 个原始震相矢量; (b1)是(a1)中震相矢量在观测坐标 $(90,0),(90,90)$ 和 $(0,0)$ 上的 3 个正交分量; (a2)同(a1), 仅第 1 个矢量 $(5,40)$ 的偏振人射角增 加 $20^{\circ}$ 成为 $(25,40)$; (b2) 是(a2)的正交分量; (a3)同(a2), 仅第 2 个震相 $(18,7)$ 的到时比(a2)中第 2 个震相到时延后了 3 个采样点; (b3)是(a3)的正交 分量. 对应颜色的垂线虚线分别标注了 3 个震相矢量到达观测台站的时间

Figure 2 The impact of arrival time and polarization of seismic waves on three-component seismograms. (a1) Three phase-vectors with polarization of incident and azimuth angle in $(5,40),(18,7)$ and $(23,23)$. (b1) The orthogonal components of vector addition of three vectors in (a1). (a2) Almost same as (a1), add $20^{\circ}$ on the incident angle of the first phase in (a1) only. (b2) The orthogonal components of vector addition of three vectors in (a2). (a3) Almost same as (a2), only add a time delay of three points (about $0.03 \mathrm{~s}$ ) on the arrival time of second phase in (a2). (b3) The orthogonal components of vector addition of three vectors in (a3). The vertical dash lines indicate the arrival time of the three phase-vectors

不仅严重影响地震波震相的记录波形, 更使依据观 测记录波形“振幅突变”或“视周期突变”的地震波震 相传统识别方法受到极大的挑战. 除初至波和强震 相外，传统震相识别方法可能带来后续震相的漏识 别或误识别. 在图2(a1) (a3)给出的3种情况中所有第 1 个(蓝色)和第 3 个(绿色)震相矢量都分别在 0.1 和 $0.41 \mathrm{~s}$ 到达观测台站, 显示 $0.41 \mathrm{~s}$ 之后没有任何新的震相到 达. 但从图2(b1) (b3)对应的各个三分量观测记录波 形上难以通过振幅突变或周期突变在 $0.41 \mathrm{~s}$ 前清晰辨 认出是否同时存在第 2 个和第 3 个震相以及它们具体
的时间起点. 相反, 在 $0.41 \mathrm{~s}$ 之后各个分量的波形上 都可以看到一定数量的振幅或周期突变. 不仅如此, 在同一组三分量记录的不同分量上振幅或周期突变 的“个数”和“时间点”不尽相同，并没有可遵循的取舍 判据.

因此，在必须面对介质各向异性和非均匀性，包 括不能排除路径非正交问题的最简单的分层均匀、各 向同性介质中，仅仅依据波形的某些“突变”特征试 图提高快-慢横波及其他二次震相、后续震相和弱震 相的到时、振幅、相位和波形等参数的测量精度是不 
客观的. 因为, 地震波震相最重要的矢量信息没有被 利用.

\section{3 改进横波分裂参数测量可靠性的途径}

介质各向异性或非均匀性引起的地震波观测走 时和理论走时的偏离、观测偏振和理论偏振的偏离以 及地震记录上震相先后到达次序的可能改变都给地 震波震相参数的测量带来了困难. 但反过来, 观测记 录上震相矢量路径和偏振的非正交关系正是震源、传 播介质和观测台站深浅部构造、变形、运动和温压状 态的综合反映.

地震学家相信三分量观测记录上的地震波携带 了关于地震震源、传播路径及观测台站下方地球介质 的完整信息. 只有突破现有在极端简化假设条件下 发展起来的、明显偏离真实介质状况的地震数据处理 框架才能走出地震横波分裂参数测量面临的困境. 这 就需要首先建立专门的地震波矢量分析方法和技术.

\section{1 三分量地震记录的矢量分析}

如前所述, 三分量地震观测记录由一系列不同 路径、不同偏振方向地震波震相矢量的非正交叠加而 成. 尽管产生路径非正交或偏振非正交的物理过程 不同, 但每一条路径上的地震波都具有各自独立的 偏振方向. 因此, 从地震波震相参数测量的角度来看 路径非正交与偏振非正交并无区别, 最终都表现为 三分量地震记录上的偏振矢量.

雷军 ${ }^{[64]}$ 曾介绍了完全基于地震波矢量特征的三 分量地震观测数据的分离想法, 给出了基于代数推 导得到的非正交地震偏振矢量分离的数学表达式, 但该代数表达式对矢量几何特征体现并不直观. 为 了更直观地展示矢量分析方法在解决非正交地震波 分离问题上的作用, 这里重新给出一种完全基于矢 量几何特征推导的非正交震相矢量分离表达式.

在三维空间中, 任意偏振方向的地震波震相矢 量都可以用该方向单位矢量 $\overrightarrow{\boldsymbol{e}}_{i}\left(\theta_{i}, \varphi_{i}\right)$ 和波形 $a_{i}(t)$ 两 个参数来表示. 其中, 波形 $a_{i}(t)$ 表示振幅随时间的变 化, 由地震震源时间过程决定. 但由于传播路径上介 质特性的差异及其对地震波的衰减耦合作用不同, 即使同一个震源路径不同的震相矢量其波形也不一 定完全相同. 如果有 3 个非共面震相矢量 $\overrightarrow{\boldsymbol{a}}_{i}$ 分别在 $t_{i}(i=1,2,3)$ 时刻先后到达观测台站, 那么正交观测坐 标系记录到的合成矢量 $\overrightarrow{\boldsymbol{G}}(t)$ 可以表示为

$$
\begin{aligned}
\overrightarrow{\boldsymbol{G}}(t) & =\overrightarrow{\boldsymbol{a}}_{1}\left(t-t_{1}\right)+\overrightarrow{\boldsymbol{a}}_{2}\left(t-t_{2}\right)+\overrightarrow{\boldsymbol{a}}_{3}\left(t-t_{3}\right) \\
& =a_{1}\left(t-t_{1}\right) \overrightarrow{\boldsymbol{e}}_{1}+a_{2}\left(t-t_{2}\right) \overrightarrow{\boldsymbol{e}}_{2}+a_{3}\left(t-t_{3}\right) \overrightarrow{\boldsymbol{e}}_{3} .
\end{aligned}
$$

对 3 个非共面震相矢量, 一定可以存在矢量 $\overrightarrow{\boldsymbol{e}}_{a}, \overrightarrow{\boldsymbol{e}}_{b}, \overrightarrow{\boldsymbol{e}}_{c}$, 分别满足:

$$
\begin{aligned}
& \overrightarrow{\boldsymbol{e}}_{a}=\overrightarrow{\boldsymbol{e}}_{2} \times \overrightarrow{\boldsymbol{e}}_{3}, \\
& \overrightarrow{\boldsymbol{e}}_{b}=\overrightarrow{\boldsymbol{e}}_{3} \times \overrightarrow{\boldsymbol{e}}_{1}, \\
& \overrightarrow{\boldsymbol{e}}_{c}=\overrightarrow{\boldsymbol{e}}_{1} \times \overrightarrow{\boldsymbol{e}}_{2} .
\end{aligned}
$$

将它们分别与观测矢量 $\overrightarrow{\boldsymbol{G}}(t)$ 点乘:

$$
\begin{aligned}
\overrightarrow{\boldsymbol{e}}_{a} \cdot \overrightarrow{\boldsymbol{G}}(t) & =\left(\overrightarrow{\boldsymbol{e}}_{2} \times \overrightarrow{\boldsymbol{e}}_{3}\right) \cdot \overrightarrow{\boldsymbol{G}}(t)=\left(\overrightarrow{\boldsymbol{e}}_{2} \times \overrightarrow{\boldsymbol{e}}_{3}\right) \cdot a_{1}\left(t-t_{1}\right) \overrightarrow{\boldsymbol{e}}_{1} \\
& =a_{1}\left(t-t_{1}\right)\left(\overrightarrow{\boldsymbol{e}}_{1} \overrightarrow{\boldsymbol{e}}_{2} \overrightarrow{\boldsymbol{e}}_{3}\right), \\
\overrightarrow{\boldsymbol{e}}_{b} \cdot \overrightarrow{\boldsymbol{G}}(t) & =\left(\overrightarrow{\boldsymbol{e}}_{3} \times \overrightarrow{\boldsymbol{e}}_{1}\right) \cdot \overrightarrow{\boldsymbol{G}}(t)=\left(\overrightarrow{\boldsymbol{e}}_{3} \times \overrightarrow{\boldsymbol{e}}_{1}\right) \cdot a_{2}\left(t-t_{2}\right) \overrightarrow{\boldsymbol{e}}_{2} \\
& =a_{2}\left(t-t_{2}\right)\left(\overrightarrow{\boldsymbol{e}}_{2} \overrightarrow{\boldsymbol{e}}_{3} \overrightarrow{\boldsymbol{e}}_{1}\right), \\
\overrightarrow{\boldsymbol{e}}_{c} \cdot \overrightarrow{\boldsymbol{G}}(t) & =\left(\overrightarrow{\boldsymbol{e}}_{1} \times \overrightarrow{\boldsymbol{e}}_{2}\right) \cdot \overrightarrow{\boldsymbol{G}}(t)=\left(\overrightarrow{\boldsymbol{e}}_{1} \times \overrightarrow{\boldsymbol{e}}_{2}\right) \cdot a_{3}\left(t-t_{3}\right) \overrightarrow{\boldsymbol{e}}_{3} \\
& =a_{3}\left(t-t_{3}\right)\left(\overrightarrow{\boldsymbol{e}}_{3} \overrightarrow{\boldsymbol{e}}_{1} \overrightarrow{\boldsymbol{e}}_{2}\right),
\end{aligned}
$$

其中矢量的混合积 $\left(\overrightarrow{\boldsymbol{e}}_{1} \overrightarrow{\boldsymbol{e}}_{2} \overrightarrow{\boldsymbol{e}}_{3}\right)=\overrightarrow{\boldsymbol{e}}_{1} \cdot\left(\overrightarrow{\boldsymbol{e}}_{2} \times \overrightarrow{\boldsymbol{e}}_{3}\right)$, 利用互换关 系 $\left(\overrightarrow{\boldsymbol{e}}_{1} \overrightarrow{\boldsymbol{e}}_{2} \overrightarrow{\boldsymbol{e}}_{3}\right)=\left(\overrightarrow{\boldsymbol{e}}_{2} \overrightarrow{\boldsymbol{e}}_{3} \overrightarrow{\boldsymbol{e}}_{1}\right)=\left(\overrightarrow{\boldsymbol{e}}_{3} \overrightarrow{\boldsymbol{e}}_{1} \overrightarrow{\boldsymbol{e}}_{2}\right)$ 将式(4)和(5)整理后, 三 个震相矢量的波形就可以表示为

$$
\begin{aligned}
& a_{1}\left(t-t_{1}\right)=\frac{\overrightarrow{\boldsymbol{e}}_{a} \cdot \overrightarrow{\boldsymbol{G}}(t)}{\left(\overrightarrow{\boldsymbol{e}}_{1} \overrightarrow{\boldsymbol{e}}_{2} \overrightarrow{\boldsymbol{e}}_{3}\right)}=\frac{\left(\overrightarrow{\boldsymbol{G}}(t) \overrightarrow{\boldsymbol{e}}_{2} \overrightarrow{\boldsymbol{e}}_{3}\right)}{\left(\overrightarrow{\boldsymbol{e}}_{1} \overrightarrow{\boldsymbol{e}}_{2} \overrightarrow{\boldsymbol{e}}_{3}\right)}, \\
& a_{2}\left(t-t_{2}\right)=\frac{\overrightarrow{\boldsymbol{e}}_{b} \cdot \overrightarrow{\boldsymbol{G}}(t)}{\left(\overrightarrow{\boldsymbol{e}}_{1} \overrightarrow{\boldsymbol{e}}_{2} \overrightarrow{\boldsymbol{e}}_{3}\right)}=\frac{\left(\overrightarrow{\boldsymbol{G}}(t) \overrightarrow{\boldsymbol{e}}_{3} \overrightarrow{\boldsymbol{e}}_{1}\right)}{\left(\overrightarrow{\boldsymbol{e}}_{1} \overrightarrow{\boldsymbol{e}}_{2} \overrightarrow{\boldsymbol{e}}_{3}\right)}, \\
& a_{3}\left(t-t_{3}\right)=\frac{\overrightarrow{\boldsymbol{e}}_{c} \cdot \overrightarrow{\boldsymbol{G}}(t)}{\left(\overrightarrow{\boldsymbol{e}}_{1} \overrightarrow{\boldsymbol{e}}_{2} \overrightarrow{\boldsymbol{e}}_{3}\right)}=\frac{\left(\overrightarrow{\boldsymbol{G}}(t) \overrightarrow{\boldsymbol{e}}_{1} \overrightarrow{\boldsymbol{e}}_{2}\right)}{\left(\overrightarrow{\boldsymbol{e}}_{1} \overrightarrow{\boldsymbol{e}}_{2} \overrightarrow{\boldsymbol{e}}_{3}\right)} .
\end{aligned}
$$

式(6) (8) 是已知观测矢量 $\overrightarrow{\boldsymbol{G}}(t)$ 和每一个震相矢量方 向 $\overrightarrow{\boldsymbol{e}}_{i}$ 时从三分量观测记录上分离获取 3 个非正交(或 正交)震相矢量完整波形和振幅的数学表达式. 其中, 分母上的混合积 $\left(\overrightarrow{\boldsymbol{e}}_{1} \overrightarrow{\boldsymbol{e}}_{2} \overrightarrow{\boldsymbol{e}}_{3}\right)$ 的值为

$$
\left(\overrightarrow{\boldsymbol{e}}_{1} \overrightarrow{\boldsymbol{e}}_{2} \overrightarrow{\boldsymbol{e}}_{3}\right)=\left|\begin{array}{ccc}
e_{1 x} & e_{1 y} & e_{1 z} \\
e_{2 x} & e_{2 y} & e_{2 z} \\
e_{3 x} & e_{3 y} & e_{3 z}
\end{array}\right| .
$$

与文献 $[64,65]$ 中非正交仿射坐标系量度系数矩 阵逆的行列式值相等: 当矢量 $\overrightarrow{\boldsymbol{e}}_{i}$ 彼此正交时 $\left(\overrightarrow{\boldsymbol{e}}_{1} \overrightarrow{\boldsymbol{e}}_{2} \overrightarrow{\boldsymbol{e}}_{3}\right)=1$, 非正交时 $\left(\overrightarrow{\boldsymbol{e}}_{1} \overrightarrow{\boldsymbol{e}}_{2} \overrightarrow{\boldsymbol{e}}_{3}\right)<1$, 分子上的混合积 与时间相关, 决定了各个震相矢量的波形. 显示某个 震相矢量的波形不是合成矢量 $\overrightarrow{\boldsymbol{G}}(t)$ 在该矢量方向的 投影, 而是在与另外两个矢量相关的某个特定方向 的投影. 如式(6)中分子 $\overrightarrow{\boldsymbol{e}}_{a} \cdot \overrightarrow{\boldsymbol{G}}(t)$ 显示矢量 $\overrightarrow{\boldsymbol{a}}_{1}$ 的波形 $a_{1}\left(t-t_{1}\right)$ 是 $\overrightarrow{\boldsymbol{G}}(t)$ 在 $\overrightarrow{\boldsymbol{e}}_{a}$ 方向的投影. 这是因为在与 $\overrightarrow{\boldsymbol{e}}_{2}$ 
和 $\overrightarrow{\boldsymbol{e}}_{3}$ 同时正交的方向 $\overrightarrow{\boldsymbol{e}}_{a}$ 上正好不会有矢量 $\overrightarrow{\boldsymbol{a}}_{2}$ 和 $\overrightarrow{\boldsymbol{a}}_{3}$ 的 投影，而只有矢量 $\overrightarrow{\boldsymbol{a}}_{1}$ 的投影. 非正交情况下 $\overrightarrow{\boldsymbol{a}}_{1}$ 和 $\overrightarrow{\boldsymbol{e}}_{a}$ 方向通常不会一致, 因此 $\overrightarrow{\boldsymbol{a}}_{1}$ 在 $\overrightarrow{\boldsymbol{e}}_{a}$ 方向上的投影振幅 总是小于其实际振幅, 分母 $\left(\overrightarrow{\boldsymbol{e}}_{1} \overrightarrow{\boldsymbol{e}}_{2} \overrightarrow{\boldsymbol{e}}_{3}\right)$ 的作用就是将 $\overrightarrow{\boldsymbol{a}}_{1}$ 的投影振幅放大还原为实际振幅. 式(7)和(8)也类似.

\section{2 三分量观测记录上地震波震相矢量的个数和 空间方向的确定}

从三分量观测数据上分离非正交震相矢量的前 提是首先确定观测记录上震相矢量的个数及其空间 方向. Lei ${ }^{[65]}$ 给出了三分量观测记录上确定震相矢量 个数的数学判据, 可从三分量观测记录的任何时间 窗内确定震相的个数, 即使震相的振幅相差 2 3个数 量级也是非常可靠的. 同时, 还给出了采用全局搜索 震相矢量空间方向的地震学准则. 其前提假设是所 有震相矢量都来自同一个震源. 但如果观测记录上 存在来自其他震源的矢量信号, 包括环境振动, 此时 基于同震震相波形相似性的全局搜索结果并不一定 唯一. 另外, 全局搜索运算效率很低, 难以满足实际 地震数据处理的需要.

三分量观测记录上包含了地震波震相矢量的方 向信息, 有多条途径有望确定矢量的方向：(1) 从时 间域波形上寻找 ${ }^{[64,65]}$, 主要基于对式(6) (8)时域波 形特征分析和代数解析来深人; (2) 一种比较直观地 获取地震波震相矢量空间方向的途经是对式(1)进行 傅里叶变换 ${ }^{[66]}$.

$$
\begin{aligned}
\operatorname{FFT}(\overrightarrow{\boldsymbol{G}}(t))= & \operatorname{FFT}\left(\overrightarrow{\boldsymbol{a}}_{1}\left(t-t_{1}\right)+\overrightarrow{\boldsymbol{a}}_{2}\left(t-t_{2}\right)+\overrightarrow{\boldsymbol{a}}_{3}\left(t-t_{3}\right)\right) \\
= & \operatorname{FFT}\left(a_{1}\left(t-t_{1}\right)\right) \overrightarrow{\boldsymbol{e}}_{1}+\operatorname{FFT}\left(a_{2}\left(t-t_{2}\right)\right) \overrightarrow{\boldsymbol{e}}_{2} \\
& +\operatorname{FFT}\left(a_{3}\left(t-t_{3}\right)\right) \overrightarrow{\boldsymbol{e}}_{3} \\
= & A_{1}(\omega) \overrightarrow{\boldsymbol{e}}_{1}+A_{2}(\omega) \overrightarrow{\boldsymbol{e}}_{2}+A_{3}(\omega) \overrightarrow{\boldsymbol{e}}_{3},
\end{aligned}
$$

其中,

$$
\begin{aligned}
& A_{1}(\omega)=\operatorname{FFT}\left(a_{1}\left(t-t_{1}\right)\right), \\
& A_{2}(\omega)=\operatorname{FFT}\left(a_{2}\left(t-t_{1}\right)\right), \\
& A_{3}(\omega)=\operatorname{FFT}\left(a_{3}\left(t-t_{1}\right)\right) .
\end{aligned}
$$

式(10)和(11)表明, 观测记录上的合成矢量 $\overrightarrow{\boldsymbol{G}}(t)$ 的傅 里叶变换可以表示为震相波形傅里叶变换的矢量和, 因为傅里叶变换是时间积分不改变地震波的空间矢 量特征. 如图3(a1) (a3)所示, 是图2(a1)中 3 个震相波 形的傅里叶振幅谱(频率半轴), 其主频分别为 23,14 和12 Hz. 图3(b1) (b3) 是图2(b1)中合成矢量 $\overrightarrow{\boldsymbol{G}}(t)$ 三
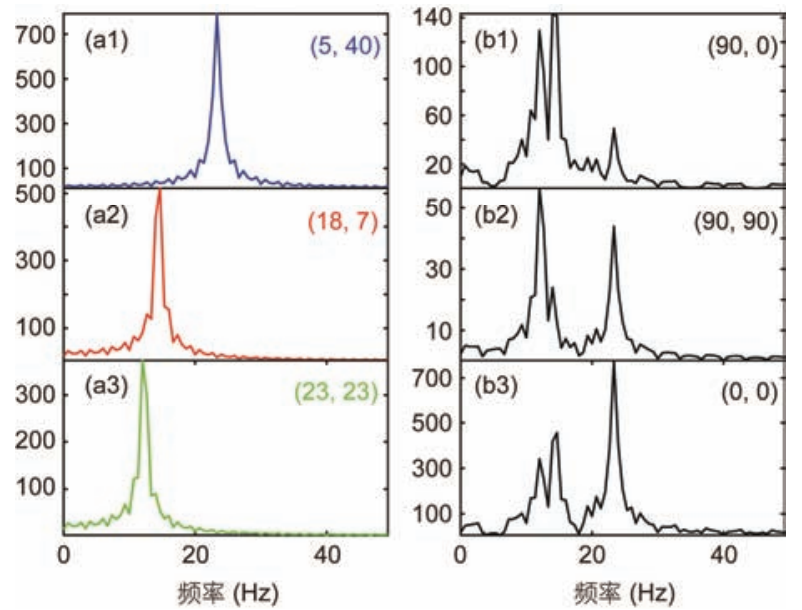

图 3 地震波矢量的傅里叶变换. (a1) (a3)为图 2(a1)中 3 个地震波震 相矢量的傅里叶振幅谱; (b1) (b3)为三分量观测记录(图 2(b1))的傅里 叶振幅谱

Figure 3 Vector analysis of seismic wave using FFT. (a1)-(a3) are the FFT of three seismic phase-vectors in Figure 2(a1). (b1)-(b3) are the FFT of three components record of seismic wave in Figure 2(b1)

个分量记录的傅里叶振幅谱，从中可以清楚地看到 在与 23,14 和 $12 \mathrm{~Hz}$ 对应的 3 个频率点附近有 3 个振幅 谱峰值，正好是图3(a1) (a3)中3个震相矢量在观测坐 标中的投影，借助这些投影分量的大小可以比较方 便地近似估算出 3 个震相矢量的空间方向，它比利用 其时域分量(图2(b1))来测量震相矢量的空间方向更 直观、效率更高.

利用式(6) (8)从观测矢量 $\overrightarrow{\boldsymbol{G}}(t)$ 还原或分离原始 3 个独立的震相矢量的波形首先需要确定各个震相矢 量的空间方向，震相矢量空间方向越准确分离得到 的波形与原始震相波形越接近. 需要注意的是，由于 图3(b1) (b3)频谱是3个原始震相的幅值谱在观测坐 标分量上相互叠加的结果，由其直接得到的震相矢 量的空间方向存在一定的误差，还需要依据具体情 况进行修正.

\section{4 讨论}

理论上对地震各向异性介质的研究已经十分广 泛，但无论物理模型如何精细，它都离不开观测数据 的检验或验证. 由于横波分裂参数测量结果的不稳 定性，特别是横波快-慢波到时差测量较大离散所凸 显出来的测量瓶颈问题, 不仅制约了从现有观测数 据中提取比以往更多更可靠信息的能力，也模糊了 未来地震各向异性研究和横波分裂观测解释的推进 
方向.

从三分量观测记录上可靠测量横波参数及其他 二次震相、后续震相和弱震相运动学和动力学参数最 突出的影响因素是实际地震记录上地震波震相矢量 广泛存在的非正交关系. 由前所述, 这种非正交关系 来源于介质的各向异性和非均匀性, 本质上是地震 波传播路径以及观测台站底部地球介质深浅构造、运 动、变形、温度和压力状态的综合反映. 因此, 从观 测记录上可靠测量非正交地震波震相矢量的参数是 了解地球内部运动和介质精细结构无法绕开的难题.

迄今为止, 数学家没有提供现成的有关矢量分
离的方法, 更没有提供三分量观测数据上可能包含 3 个以上震相矢量的数学解析途径. 因此, 横波快波偏 振、快-慢波到时差以及地震记录上其他后续震相、 弱震相、转换震相的准确测量都受到了极大的限制. 唯一可能改善这种状况的途径是直接针对三分量观 测数据总地震波震相的矢量特征, 建立独立的地震 波矢量分析方法.

只要重视介质复杂性对地震纵横波观测带来的 影响、充分了解实际三分量地震记录上地震波的基本 特征、不断发展和完善矢量分析技术就有可能推进横 波观测解释取得实质性的进展.

致谢感谢挪威DNO石油公司首席地球物理学家叶三余博士对全文的审阅和中英文摘要的修改.

\section{参考文献}

1 Crampin S. Seismic-wave propagation through a cracked solid: Polarization as a possible dilatancy diagnostic. Geophys J Int, 1978, 53: 467-496

2 Crampin S, Evans R, Üçer B, et al. Observations of dilatancy-induced polarization anomalies and earthquake prediction. Nature, 1980, 286: $874-877$

3 Crampin S. A review of wave motion in anisotropic and cracked elastic-media. Wave Motion, 1981, 3: 343-391

4 Crampin S. Effective anisotropic elastic constants for wave propagation through cracked solids. Geophys J Int, 1984, 76: 135-145

5 Crampin S. Evaluation of anisotropy by shear-wave splitting. Geophysics, 1985, 50: 142-152

6 Crampin S, Booth D C. Shear-wave polarizations near the North Anatolian Fault-II. Interpretation in terms of crack-induced anisotropy. Geophys J Int, 1985, 83: 75-92

7 Thomsen L. Weak elastic anisotropy. Geophysics, 1986, 51: 1954-1966

8 Zoback M D, Zoback M L, Mount V S, et al. New evidence on the state of stress of the San Andreas Fault system. Science, 1987, 238: 1105-1111

9 Kaneshima S, Ando M, Crampin S. Shear-wave splitting above small earthquakes in the Kinki district of Japan. Phys Earth Planet Inter, 1987, 45: 45-58

10 Kaneshima S, Ando M, Kimura S. Evidence from shear-wave splitting for the restriction of seismic anisotropy to the upper crust. Nature, 1988, 335: 627-629

11 Yao C, Wang P D, Chen Y T. Shear-wave polarization and crack induced anisotropy of upper crust in Lulong North China (in Chinese). Chin J Geophys, 1992, 35: 305-315 [姚陈, 王培德, 陈运泰. 卢龙地区 S 波偏振与上地壳裂隙各向异性. 地球物理学报, 1992, 35: 305-315]

12 Ding Z F, Zeng R S. Observation and study of shear wave anisotropy in Tibetan Plateau (in Chinese). Chin J Geophys, 1996, 39: 211-220 [丁志峰, 曾融生. 青藏高原横波分裂的观测研究. 地球物理学报, 1996, 39: 211-220]

13 Lei J, Wang P D, Yao C, et al. The near-field shear wave splitting and its relation with structure in Jianchuan, Yunnan Province (in Chinese). Chin J Geophys, 1997, 40: 791-801 [雷军, 王培德, 姚陈, 等. 云南剑川近场横波特征及其与构造的关系. 地球物理学报, 1997, 40: 791-801]

14 Gao Y, Zheng S H, Zhou H L. Polarization patterns of fast shear wave in Tangshan region and their variations (in Chinese). Chin J Geophys, 1999, 42: 228-232 [高原, 郑斯华, 周萬兰. 唐山地区快剪切波偏振图像及其变化. 地球物理学报, 1999, 42: 228-232]

15 Ding Z F, Wu Y, Wang H, et al. The variation features of shear-wave splitting in the source region of Wenchuan earthquake in 2008 (in Chinese). Sci Sin Terr, 2008, 38: 1600-1604 [丁志峰, 武岩, 王辉, 等. 2008 年汶川地震震源区横波分裂的变化特征. 中国科学 D 辑: 地球科学, 2008, 38: 1600-1604]

16 Chang L J, Wang C Y, Ding Z F, et al. Seismic anisotropy of upper mantle in the northeastern margin of the Tibetan Plateau (in Chinese). Chin J Geophys, 2008, 51: 431-438 [常利军, 王椿镛, 丁志峰, 等. 青藏高原东北缘上地幔各向异性研究. 地球物理学报, 2008, 51: 431-438] 
17 Gao Y, Wang Q, Zhao B, et al. A rupture blank zone in middle south part of Longmenshan Faults: Effect after Lushan $M_{\mathrm{s}} 7.0$ earthquake of 20 April 2013 in Sichuan (in Chinese). Sci Sin Terr, 2013, 43: 1038-1046 [高原, 王琼, 赵博, 等. 龙门山断裂带中南段的一个破裂空 段——芦山地震的震后效应. 中国科学 D 辑: 地球科学, 2013, 43: 1038-1046]

18 Ando M, Ishikawa Y, Yamazaki F. Shear wave polarization anisotropy in the upper mantle beneath Honshu, Japan. J Geophysl Res-Solid Earth, 1983, 88: 5850-5864

19 Barruol G L, Kern H. Seismic anisotropy and shear wave splitting in lower-crustal and upper-mantle rocks from the Ivera Zone's experimental and calculated data. Phys Earth Planet Inter, 1996, 95: 175-194

20 Vinnik L P, Kosarev G L, Makeyeva L I. Lithospheric anisotropy as indicated by SKS and SKKS waves. Doklady Earth Sci Sect, 1984, 278: $39-43$

21 Silver P G, Chan W W. Shear wave splitting and subcontinental mantle deformation. J Geophys Res-Solid Earth, 1991, 96: 16429-16454

22 Gao S, Davis P M, Liu H, et al. SKS splitting Beneath continental rift zones. J Geophys Res, 1997, 102: 22781-22797

23 Restivo A, Helffrich G. Core-mantle boundary structure investigated using SKS and SKKS polarization anomalies. Geophys J Int, 2006, 165: 288-302

24 Wang Y, Wen L. Complex seismic anisotropy at the border of a very low velocity province at the base of the Earth's mantle. J Geophys Res-Solid Earth, 2007, 112: B09305, doi: 10.1029/2006JB004719

25 He Y M, Wen L X. Geographic boundary of "Pacific Anomaly" and its geometry and transitional structure in the north. J Geophys Res, 2012, 117: B09308, doi: 10.1029/2012JB009436

26 Feng L L, Chen Y T, Lei J. SKS splitting analysis of seismic anisotropy beneath Mid-Eastern Africa (in Chinese). Acta Seismol Sin, 2014, 36: 981-996 [冯力理, 陈运泰, 雷军. 通过 SKS 分裂分析非洲中东部地区地震各向异性. 地震学报, 2014, 36: 981-996]

27 Song X, Helmberger D V. Anisotropy of Earth's inner core. Geophys Res Lett, 1993, 20: 2591-2594

28 Song X D, Richards P G. Seismological evidence for differential rotation of the Earth's inner core. Nature, 1996, 382: 221-224

29 Liu B, Zhang Q S, Wang B S, et al. The Velocity anisotropy $y$ of seismic wave in earth inner core is related to the earth rotation (in Chinese). Chin Sci Bull, 1999, 44: 1209-1211 [刘斌, 张群山, 王宝善, 等. 地球内核的地震波速各向异性与其自转有关. 科学通报, 1999, 44: 1209-1211]

30 Booth D C, Crampin S, Lovell J H, et al. Temporal changes in shear wave splitting during an earthquake swarm in Arkansas. J Geophys Res-Solid Earth, 1990, 95: 11151-11164

31 Crampin S. Geological and industrial implications of extensive-dilatancy anisotropy. Nature, 1987, 328: 491-496

32 Crampin S. Implications of temporal changes in shear-wave splitting. In: SEG Technical Program Expanded Abstracts 1988. Anaheim: Society of Exploration Geophysicists, 1988. 1142-1144

33 Peacock S, Crampin S, Booth D C, et al. Shear wave splitting in the Anza seismic gap, southern California: Temporal variations as possible precursors. J Geophys Res-Solid Earth, 1988, 93: 3339-3356

34 Crampin S, Booth D C, Evans R, et al. Changes in shear wave splitting at Anza near the time of the North Palm Springs Earthquake. J Geophys Res-Solid Earth, 1990, 95: 11197-11212

35 Bush I, Crampin S. Paris Basin VSPs: Case history establishing combinations of fine-layer (or lithologic) anisotropy and crack anisotropy from modelling shear wave fields near point singularities. Geophys J Int, 1991, 107: 433-447

36 MacBeth C. Inverting shear-wave polarizations for anisotropy using three-component offset VSPs: Synthetic seismograms. Geophys J Int, 1991, 107: 571-583

37 MacBeth C, Wild P, Crampin S, et al. Optimal VSP geometry for recording shear-wave anisotropy. In: 55th East Asian Economic Group (EAEG) Meeting, 1993

38 Zhang X L, Zhang F, Li X Y, et al. The influence of hydraulic fracturing on velocity and microseismic location (in Chinese). Chin J Geophys, 2013, 56: 3552-3560 [张晓林, 张峰, 李向阳, 等. 水力压裂对速度场及微地震定位的影响. 地球物理学报, 2013, 56: 3552-3560]

39 Li G F, Peng S P, He B S. Use a technique of P-wave azimuthal amplitude variation with offset to detect coal fracture in 3D seismic exploration (in Chinese). Chin Sci Bull, 2005, 50(s1): 138-142 [李国发, 彭苏萍, 何兵寿, 等. 利用三维地震勘探纵波方位振幅随偏移 距变化技术检测煤田裂隙. 科学通报, 2005, 50(s1): 138-142]

40 Yang D Y, Wang Y, Zhang M G. The wave field characteristics of the fracture weakness parameter (in Chinese). Chin J Geophys, 2011, 54: 862-866 [杨德义, 王望, 张美根. 裂隙弱度参数的波场特征. 地球物理学报, 2011, 54: 862-866]

41 Yao C, Hao C T, Zhang G L. A study of synthetic seismograms for SKS-wave response to crustal fractured-induce anisotropy (in Chinese). Chin J Geophys, 2016, 59: 2498-2509 [姚陈, 郝重涛, 张广利. SKS 波对地壳裂隙各向异性的响应一一理论地震图研究. 地球 物理学报, 2016, 59: 2498-2509]

42 Liu Y, Crampin S, Main I. Shear-wave anisotropy: Spatial and temporal variations in time delays at Parkfield, Central California. Geophys J Int, 1997, 130: 771-785 
43 Crampin S, Zatsepin S V. Modelling the compliance of crustal rock-II. Response to temporal changes before earthquakes. Geophys J Int, 1997, 129: 495-506

44 Angerer E, Crampin S, Li X Y, et al. Processing, modelling and predicting time-lapse effects of over pressured fluid-injection in a fractured reservoir. Geophys J Int, 2002, 149: 267-280

45 Crampin S, Lovell J H. A decade of shear-wave splitting in the Earth's crust: What does it mean? What use can we make of it? And what should we do next? Geophys J Int, 1991, 107: 387-407

46 Crampin S, Peacock S. A review of the current understanding of seismic shear-wave splitting in the Earth's crust and common fallacies in interpretation. Wave Motion, 2008, 45: 675-722

47 Crampin S, Gao Y. A review of techniques for measuring shear-wave splitting above small earthquakes. Phys Earth Planet Int, 2006, 159: $1-14$

48 Aster R C, Shearer P M, Berger J. Quantitative measurements of shear wave polarizations at the Anza seismic network, southern California: Implications for shear wave splitting and earthquake prediction. J Geophys Res-Solid Earth, 1990, 95: 12449-12473

49 Crampin S, Volti T, Stefánsson R. A successfully stress-forecast earthquake. Geophys J Int, 1999, 138: F1-F5

50 Crampin S. The fracture criticality of crustal rocks. Geophys J Int, 1994, 118: 428-438

51 Booth D C, Crampin S. The anisotropic reflectivity technique: Theory. Geophys J Int, 1983, 72: 755-766

52 Taylor D B. Double contour integration for transmissions from point sources through anisotropic layers as used in ROCPAC software. Geophys J Int, 1987, 91: 373-381

53 Fryer G J, Frazer L N. Seismic waves in stratified anisotropic media. Geophys J Int, 1984, 78: 691-710

54 Fryer G J, Frazer L N. Seismic waves in stratified anisotropic media-II. Elastodynamic eigensolutions for some anisotropic systems. Geophys J Int, 1987, 91: 73-101

55 Silver P G. Seismic anisotropy beneath the continents: Probing the depths of geology. Ann Rev Earth Planet Sci, 1996, 24: 385-432

56 Wookey J, Kendall J M. Evidence of midmantle anisotropy from shear wave splitting and the influence of shear-coupled P waves. J Geophys Res, 2004, 109: 253-258

57 Johnson P A, Rasolofosaon P N J. Nonlinear elasticity and stress-induced anisotropy in rock. J Geophys Res, 1996, 101: 3113-3124

58 Crampin S, McGonigle R. The variation of delays in stress-induced anisotropic polarization anomalies. Geophys J R Astr Soc, 1981, 64: 115-131

59 Rüger A. Variation of P-wave reflectivity with offset and azimuth in anisotropic media. Geophysics, 1998, 63: 935-947

60 Crampin S. A review of the effects of crack geometry on wave propagation through aligned cracks. Can J Expl Geophys, 1993, 29: 3-17

61 Li X Y, Crampin S. Linear-transform techniques for processing shear-wave anisotropy in four-component seismic data. Geophysics, 1993, 58: $240-256$

62 Li X Y, MacBeth C, Crampin S. Interpreting non-orthogonal split shear waves for seismic anisotropy in multicomponent VSPS. Geophys Prospect, 1998, 46: 1-27

63 Yao C, Lei J, Xiong Y W. The effects of weak crack induced anisotropy on seismic body waves (in Chinese). Earthq Res China, 1997, 13: 232-240 [姚陈，雷军，熊杨武. 弱裂隙各向异性对地震体波的效应. 中国地震, 1997, 13: 232-240]

64 Lei J. Separation and reconstruction of three-component seismic wave vector field. Doctor Dissertation. Beijing: Institute of Geology, China Earthquake Administration, 1999 [雷军. 三分量地震矢量波场的分离与重建. 博士学位论文. 北京: 中国地震局地质研究所, 1999]

65 Lei J. A method for non-orthogonal seismic polarization-vector separation. Geophys J Int, 2005, 162: 965-974

66 Lei J. The vector signal processing method and its vector signal processing system. PRC Patent, ZL 2007 1 0080303.9, 2007-02-27 [雷军. 矢量信号处理方法及采用该方法的矢量信号处理系统的制作方法.中国专利, ZL 2007 0080303.9, 2007-02-27] 


\section{Impact of anisotropic and inhomogeneous medium on measuring seismic shear-wave splitting}

\section{LEI Jun}

School of Earth and Space Sciences, Peking University, Beijing 100871, China

E-mail: leij@pku.edu.cn

Parameters of seismic shear-wave splitting are important basis for determining the features of anisotropy in earth medium. Significant scatters due to inaccuracy in measuring polarization and time delay of fast-slow shear waves were major obstacles in seismic anisotropy and shear-wave splitting study in the past decades. This paper analyses the following points: (1) difference of seismic wave propagation characteristics in anisotropic medium compared with isotropic medium; (2) non-orthogonal nature of S-waves in anisotropic medium; (3) non-orthogonal relationship of S- to P-waves due to different travel paths. Hereby it makes clear that the anisotropy and inhomogeneity of real earth medium can lead to differences in traveltime, polarization and phase sequence of observed seismogram in comparison to those of theoretical calculation that is based on isotropic medium assumption. Furthermore, velocity variations due to anisotropy and inhomogeneity, different travel paths due to different wave types can lead to observation of wave arrivals of different polarization within a very small time window, making non-orthogonality a common problem in a wide range of seismic recordings. In consequence, non-orthogonality due to either different polarization or/and different travel paths impedes the traditional orthogonal wave separation techniques from extracting accurate shear wave splitting parameters, particularly the travel time delay between fast and slow shears waves, from $3 \mathrm{C}$ seismic record, thus causing significant scatter of the measured results.

This paper also analyses challenges in current techniques of seismic phase identification and parameter determination. Despite decade-long development, methods in shear wave analysis and parameter extraction are largely based on orthogonal wave separation techniques that are valid only for homogenous and isotropic medium. To reduce the scatter and improve the accuracy of measured shear wave splitting parameter, the vector nature of seismic phases and their non-orthogonal relationship must be taken into account. Using synthetic data, this paper illustrates: (1) non-uniqueness of orthogonal wave field separation; (2) ambiguity/uncertainty of waveform for vector phases on observed seismic records; (3) failure and/or error in identification of seismic phases by traditional seismic phase and waveform analysis methods.

It proposes approaches to improve reliability in estimating shear wave splitting parameters. Here it introduces: (1) definition of polarization direction and number of seismic vector phases on 3C seismic record; (2) vector analysis method for non-orthogonal seismic vector phases. Using the above methodology, the paper uses an algorithm example to show how to extract complete waveform under certain assumptions. Hereby the formulas of wave field separation expressed in unit vector of the polarization direction are derived. The Fourier transform of the formulas and its projection on different coordinate axis can be used as rough estimates for directions of observed seismic vector phases.

With the current methods, it is impossible to know whether a 3C seismic record is free from influences of anisotropy or inhomogeneity origins; neither can we determine quantitatively their respective influences.

The paper stresses that non-orthogonality is an inherent nature of common $3 \mathrm{C}$ seismic recordings, and it is a significant factor for reliable determination of kinematic and dynamic parameters of seismic phases, including splitted shear waves, secondary and later weak arrivals. The non-orthogonal relationship among seismic phases is a complex expression, not only influenced by anisotropy and inhomogeneity, but also controlled by local geology under recording stations and by physical states like stress, deformation, temperature and pressure of the medium along wave paths. The successful separation of all different seismic phases should deal with their vector nature, thus improving our understanding of observed seismic waves.

shear wave splitting, anisotropy, inhomogeneity, non-orthogonality, vector

doi: 10.1360/N972016-01470 